\title{
Capacity Study of Vehicle-to-Roadside MIMO Channels with a Line-of-Sight Component
}

\author{
Michail Matthaiou*, David I. Laurenson*, and Cheng-Xiang Wang ${ }^{\dagger}$ \\ *Institute for Digital Communications, Joint Research Institute for Signal and Image Processing, \\ School of Engineering and Electronics, The University of Edinburgh, \\ Mayfield Road, EH9 3JL, Edinburgh, U.K. \\ Email: \{M.Matthaiou, Dave.Laurenson\}@ed.ac.uk \\ $\dagger$ Joint Research Institute for Signal and Image Processing, \\ School of Engineering and Physical Sciences, Heriot-Watt University \\ EH14 4AS, Edinburgh, U.K. \\ Email: cheng-xiang.wang@hw.ac.uk
}

\begin{abstract}
In this paper, the performance of a Multiple-Input Multiple-Output (MIMO) system is assessed in the case of vehicle-to-roadside communications. We investigate a Line-ofSight $(\mathrm{LoS})$ scenario where a specular wavefront impinges on the receive side. Under these conditions, the channel response is usually rank deficient due to the high correlation between the spatial LoS responses, unless specific antenna geometries are employed in order to achieve subchannel orthogonality. For our investigation, a recently proposed criterion, which maximizes the LoS-channel rank and eventually the channel capacity, is revised for different array configurations.
\end{abstract}

\section{INTRODUCTION}

The automotive industry strives decisively in the direction of advanced active safety. On these grounds, the so-called Dedicated Short Range Communications (DSRC) protocol has been developed to be used for vehicle-to-vehicle and vehicle-to-roadside communications. It is planned to work in parallel with cellular communications by providing high data rates with a low latency in short range propagation scenarios. The official spectrum allocation for DSRC was completed in 1999 by the U.S. Federal Communication Commission which designated $75 \mathrm{MHz}$ of bandwidth at the $5.9 \mathrm{GHz}$ Intelligent Transportation System (ITS) frequency band; it is also being standardized as the IEEE 802.11p protocol [1]. Some of the potential DSRC applications include local danger warning, vehicle collision avoidance, traffic control and remote toll collection. In addition, a plethora of more demanding applications, such as high-speed networking, video streaming and mobile commerce, are underway. These mandate the use of efficient technologies, such as MIMO, which can offer an enhanced spectral efficiency by deploying multiple antenna elements at both ends of the communication link.

In our investigation, we merely consider a vehicle-toroadside scenario where the transmitter $(\mathrm{Tx})$ is mounted on a fixed infrastructure unit while the receiver $(\mathrm{Rx})$ is placed on a car which moves on a motorway. In such a case, the presence of a LoS component is highly likely especially if the traffic on the motorway is sparse and hence there are no obstacles between the two ends.
The case of LoS propagation is usually thought to limit the beneficial effects of MIMO technology which were initially demonstrated in the breakthrough work of Foschini and Gans [2]. This can be attributed to the increased correlation, which reduces the rank of the channel matrix thereby resulting in a lower capacity, between the received signals [3]-[5]. A feasible solution in order to mitigate these undesired effects lies in the use of specifically designed antenna arrays [6]-[8]. In particular, the orthogonality between the spatial subchannels, which is a key condition for capacity maximization, can be achieved as long as special antenna geometries are employed at both the Tx and the $\mathrm{Rx}$ [9].

In this paper, we revise one of the recently developed criteria for the case of DSRC and thereafter we investigate in depth the performance of the proposed scheme. The remainder of this paper is organized as follows: In Section II, the channel model employed throughout the paper is outlined. In Section III, the criteria for maximizing the LoS MIMO capacity are derived in a straightforward manner. In Section IV, a detailed sensitivity study is provided by means of capacity variations as a function of different parameters. Finally, Section V concludes the paper and summarizes the key findings.

\section{Channel Model AND CAPACITy FORMUla}

We consider a MIMO system equipped with $N_{r}$ elements at the receive side and $N_{t} \geq N_{r}$ elements at the transmit side. For such a system, the complex input-output relationship can be written for the discrete case as

$$
\mathbf{y}=\mathbf{H x}+\mathbf{n}
$$

where $\mathbf{x} \in \mathbb{C}^{N_{t} \times 1}$ is the transmitted signal vector, $\mathbf{y} \in \mathbb{C}^{N_{r} \times 1}$ is the noise-corrupted received signal and $\mathbf{n} \in \mathbb{C}^{N_{r} \times 1}$ corresponds to the additive AWGN noise which is usually modeled as $\mathbb{C} \mathcal{N}\left(0, \sigma_{n}^{2}\right)$. The term $\mathbf{H} \in \mathbb{C}^{N_{r} \times N_{t}}$ is widely referred to as the channel transfer function matrix and contains the complex responses between each pair of transmit and receive antennas. In fact, it can describe effectively a MIMO channel given a specific antenna configuration and bandwidth [10]. 
In the case of LoS propagation, the channel matrix $\mathbf{H}$ consists of a spatially deterministic specular component $\mathbf{H}_{\mathrm{L}}$, which contains the free-space responses and a randomly distributed component $\mathbf{H}_{\mathrm{W}}$ which accounts for the scattered signals. Then, the channel model reads as [11]

$$
\mathbf{H}=\sqrt{\frac{K}{K+1}} \mathbf{H}_{\mathrm{L}}+\sqrt{\frac{1}{K+1}} \mathbf{H}_{\mathrm{W}}
$$

where $K$ denotes the Ricean $K$-factor expressing the ratio of powers of the free-space signal and the scattered waves. The entries of $\mathbf{H}_{\mathrm{L}}$ represent essentially spherical waveforms and therefore are of the complex form $e^{-j k d_{m, n}} / d_{m, n}$ where $k=2 \pi / \lambda$ is the wavenumber corresponding to the carrier wavelength $\lambda$ and $d_{m, n}$ is the distance between a receive element $m \in\left\{1,2, \ldots, N_{r}\right\}$ and a transmit element $n \in$ $\left\{1,2, \ldots, N_{t}\right\}$. If we assume that the differences in path losses are negligible, the free-space channel matrix can be succinctly expressed as ${ }^{1}$

$$
\mathbf{H}_{\mathbf{L}}=\left[\begin{array}{cccc}
e^{-j k d_{1,1}} & e^{-j k d_{1,2}} & \cdots & e^{-j k d_{1, N_{t}}} \\
e^{-j k d_{2,1}} & \ddots & & \vdots \\
\vdots & & & \vdots \\
e^{-j k d_{N_{r}, 1}} & \cdots & & e^{-j k d_{N_{r}, N_{t}}}
\end{array}\right] .
$$

For a purely deterministic channel $\left(\mathbf{H}=\mathbf{H}_{\mathrm{L}}\right)$, the capacity is given by the following formula (assuming perfect channel state information at the Rx but no knowledge at the Tx) [2]

$$
C=\log _{2}\left(\operatorname{det}\left(\mathbf{I}_{N_{r}}+\frac{\rho}{N_{t}} \mathbf{H H}^{\mathrm{H}}\right)\right)
$$

where $\mathbf{I}_{N_{r}}$ is the $N_{r} \times N_{r}$ identity matrix, $\rho$ is the Signal-toNoise Ratio (SNR) per receiver branch while $(\cdot)^{\mathrm{H}}$ expresses the Hermitian transposition. For $N_{r}>N_{t}$, the product $\mathbf{H H}^{\mathrm{H}}$ should be replaced by $\mathbf{H}^{\mathrm{H}} \mathbf{H}$ and $\mathbf{I}_{N_{r}}$ with $\mathbf{I}_{N_{t}}$. We note that the above capacity formula relies on a uniform power allocation scheme at all transmit elements. In order to remove the effects of the average SNR on the channel capacity, we normalize the channel response so that the constraint

$$
E\left\{\|\mathbf{H}\|_{F}^{2}\right\}=N_{r} N_{t}
$$

is fulfilled. The term $\|\cdot\|_{F}$ corresponds to the Frobenius norm of a matrix.

\section{MAXIMUM LOS MIMO CAPACITY CRITERIA}

For sake of simplicity, we consider the special case of $N_{r}=N_{t}=2$. Similar conclusions can be drawn for larger geometries with trivial manipulations. In this case, the capacity is maximized when $\mathbf{H H}^{\mathrm{H}}=2 \mathbf{I}_{2}$. That is, all eigenvalues of $\mathbf{H H}^{\mathrm{H}}$ become equal and therefore we end up with perfectly orthogonal MIMO spatial subchannels. Then, the maximum capacity is given in a closed-form expression as $C_{\max }=N_{r} \log _{2}(1+\rho)$. In [7], it was shown that this condition is satisfied when

$$
\left|d_{1,1}-d_{2,1}+d_{2,2}-d_{1,2}\right|=(2 r+1) \frac{\lambda}{2}, \quad r \in \mathbf{Z}^{+}
$$

\footnotetext{
${ }^{1}$ We have assumed, without loss of generality, isotropic radiators.
}

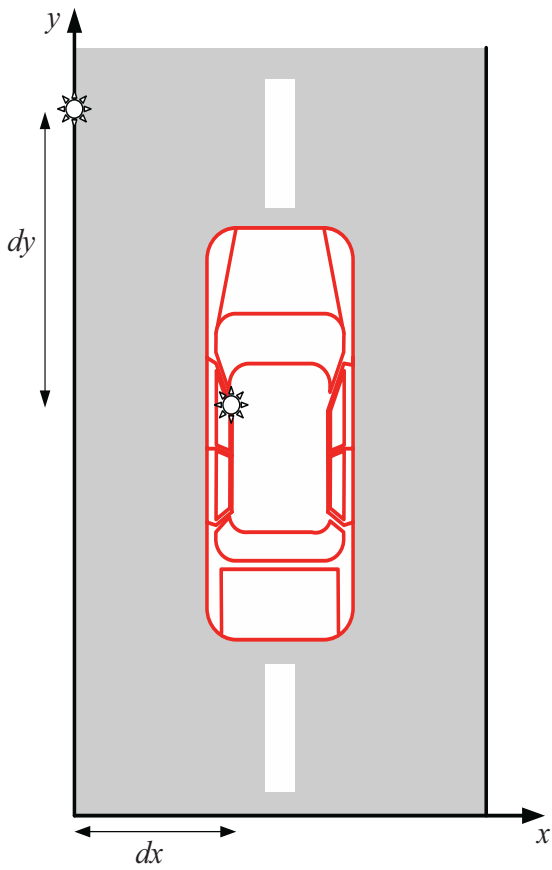

Fig. 1. Top view of a vehicle-to-roadside propagation scenario.

where $\mathbf{Z}^{+}$is the set of positive integers. In physical terms, the authors therein concluded that the sum of path differences $\left(d_{1,1}-d_{2,1}\right)$ and $\left(d_{2,2}-d_{1,2}\right)$ needs to be an odd integer multiple of a half wavelength. Clearly, we have expressed the capacity criterion as a function of distances between the antenna elements. Unfortunately, this expression is impractical since it disregards the array geometries and orientations.

A more tractable criterion can be derived by considering the geometry depicted in Fig. 1 which is basically a top view of the propagation scenario under consideration where the car is moving on a straight trajectory along the $y$-axis and both arrays are placed perpendicular to the ground. The distance between the two arrays on the $x$ and $y$ axes are respectively $d x$ and $d y$ whereas the constant height difference is given as $d z$.

In order to obtain a better understanding we have plotted a side view of the same scenario in Fig. 2. An axis rotation by an angle $\theta$ has been conducted around the $y$-axis in order to make the array origins lie on the same axis and ease the post-processing. The coordinates of all elements with regard to this new coordinate system $x^{\prime} y z^{\prime}$ are also included in the graph. We have assumed that both ends employ uniform linear arrays (ULAs) with the inter-element distances being $s_{1}$ at the Tx and $s_{2}$ at the Rx respectively.

The distance between the first element of each array is

$$
D=\sqrt{d x^{2}+d y^{2}+d z^{2}}
$$

while the rotation angle $\theta$ can be defined as

$$
\cos \theta=\frac{\sqrt{d x^{2}+d y^{2}}}{D}
$$




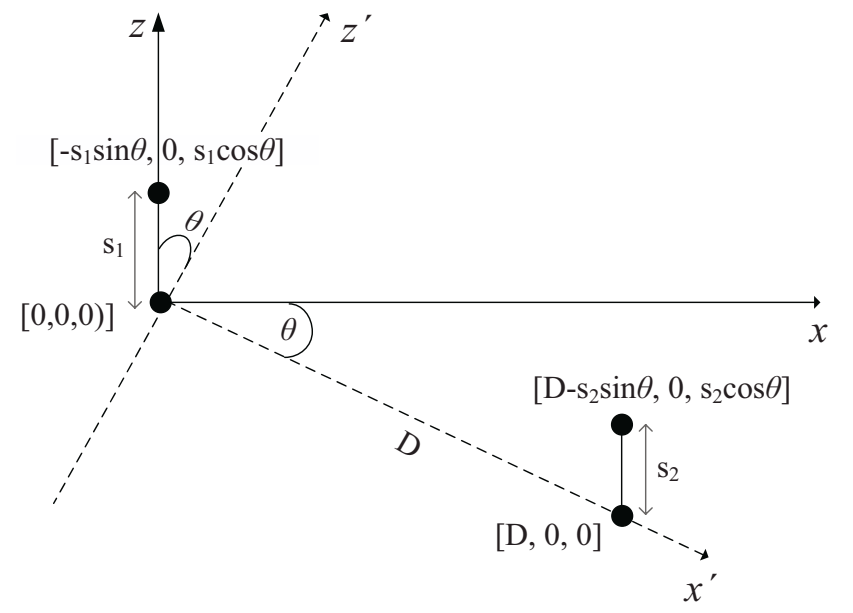

Fig. 2. Side view of a vehicle-to-roadside propagation scenario with 2element ULAs at both ends.

$$
\sin \theta=\frac{d z}{D}
$$

The Euclidean distances between all antenna pairs are given by

$$
\begin{array}{r}
d_{1,1}=D \\
d_{1,2}=\sqrt{\left(D+s_{1} \sin \theta\right)^{2}+\left(s_{1} \cos \theta\right)^{2}} \\
d_{2,1}=\sqrt{\left(D-s_{2} \sin \theta\right)^{2}+\left(s_{2} \cos \theta\right)^{2}} \\
d_{2,2}=\sqrt{\left(D+\left(s_{1}-s_{2}\right) \sin \theta\right)^{2}+\left(\left(s_{1}-s_{2}\right) \cos \theta\right)^{2}} .
\end{array}
$$

As a next step, we introduce a Taylor series approximation in order to simplify the above set of equations

$$
\begin{aligned}
\sqrt{(D+\mu)^{2}+\nu^{2}} & =(D+\mu) \sqrt{1+\frac{\nu^{2}}{(D+\mu)^{2}}} \\
& \approx(D+\mu)+\frac{\nu^{2}}{2(D+\mu)} .
\end{aligned}
$$

This approach is sufficiently accurate as long as $(D+\mu)^{2} \gg$ $\nu$; this holds true for all practical systems where $D$ is of order of tens of meters while $s_{1}, s_{2}$ are typically of order of centimeters. Then, we directly get

$$
\begin{array}{r}
d_{1,1}=D \\
d_{1,2} \approx D+s_{1} \sin \theta+\frac{\left(s_{1} \cos \theta\right)^{2}}{2\left(D+s_{1} \sin \theta\right)} \\
d_{2,1} \approx D-s_{2} \sin \theta+\frac{\left(s_{2} \cos \theta\right)^{2}}{2\left(D-s_{2} \sin \theta\right)} \\
d_{2,2} \approx D+\left(s_{1}-s_{2}\right) \sin \theta+\frac{\left(\left(s_{1}-s_{2}\right) \cos \theta\right)^{2}}{2\left(D+\left(s_{1}-s_{2}\right) \sin \theta\right)} .
\end{array}
$$

A further simplification is easily achieved if we approximate the denominators in (16)-(18) by $2 D$. Once more, the error introduced is negligible. By replacing (15) and modified (16)(18) into (6), the simplified maximum criterion can be written as

$$
s_{1} s_{2} \approx \lambda\left(r+\frac{1}{2}\right) \frac{D}{\cos ^{2} \theta}, \quad r \in \mathbf{Z}^{+} .
$$

It is noteworthy that this formula is a function of the interelement distances, the Tx-Rx distance, the orientation of the arrays and the carrier frequency. In general, larger distance requires larger antenna spacings while higher frequency requires smaller antenna spacings. For fixed frequency and Tx-Rx distance, the arrays can be easily designed so that subchannel orthogonality is attained.

\section{CAPACITY VARIATIONS}

While the aforementioned criterion is simple it does not take into account possible deviations from the optimum values which are always present in realistic propagation conditions due to either design inaccuracies or positioning displacements. In light of this fact, we address next the performance of the proposed scheme with a view to the variations of the factors that affect it.

\section{A. Displacement effects}

For the scenario under investigation, the receive array is not fixed at a specific position but its location changes with the car motion. We firstly evaluate the performance of the proposed scheme by means of displacement from an optimal point within an area of interest. We assume the optimal point to occur when $d x=5 \mathrm{~m}, d y=0$ and $d z=2 \mathrm{~m}\left(D_{\text {opt }}=5.39\right.$ $\mathrm{m}$ and $\theta_{\mathrm{opt}}=21.8^{\circ}$ ). The carrier frequency is $5.9 \mathrm{GHz}$ and the SNR $\rho$ was set equal to $20 \mathrm{~dB}$. Using the solution of equal inter-element spacings, the minimum optimal spacing for the full-rank model (i.e. the solution of (19 for $r=0$ ) gives $s_{1}=s_{2}=39.85 \mathrm{~cm}$. At this point, capacity reaches its maximum value which is $C_{\max }=13.32 \mathrm{bits} / \mathrm{sec} / \mathrm{Hz}$.

We examine the sensitivity of the proposed orientation to displacements from the optimal point on the $x-y$ plane. The considered area is determined as $0 \leq D_{x} \leq 15 \mathrm{~m}$ and $-50 \leq$ $D_{y} \leq 50 \mathrm{~m}$ where $D_{x}, D_{y}$ are the displacements on the corresponding axes. The variations of capacity are illustrated in Fig. 3. We can easily note that system capacity is highly sensitive to displacements in the direction of $y$-axis whereas it seems to be less susceptible to displacements (smaller dynamic range) in the direction of $x$-axis. As anticipated, when the car is passing by the roadside $\left(D_{y}=0\right)$ the largest capacity values are observed. For the range $-20 \leq D_{y} \leq 20 \mathrm{~m}$, the proposed scheme delivers high capacities and outperforms the common identically and independent distributed (i.i.d) Rayleigh system which yields an ergodic capacity of $11.4 \mathrm{bits} / \mathrm{sec} / \mathrm{Hz}$ for the same operating SNR.

\section{B. Scattering effects}

So far, we have assumed that free-space propagation is the only mechanism present within the communication channel. In practice though, some degree of scattering is always present due to the interaction of the transmitted signal with the physical environment as it travels toward the Rx. Hence, it is of vital importance to study the effects of scattering on 


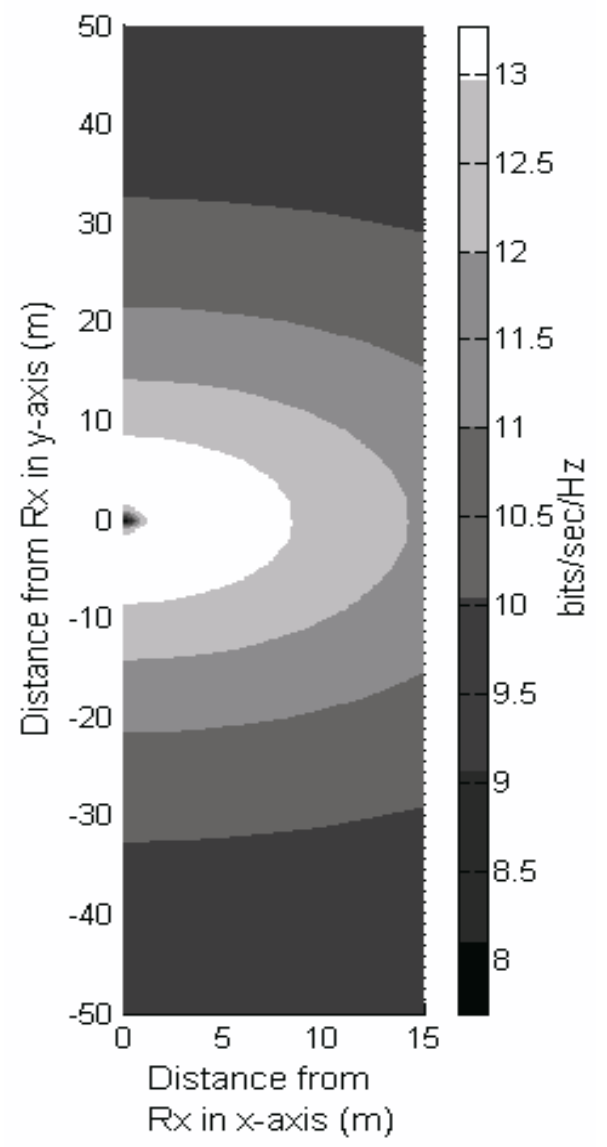

Fig. 3. Capacity variation against displacement on the $x-y$ plane.

capacity. We refer back to (2) which is essentially a stochastic model taking into account local scattering. For a stochastic MIMO channel, the ergodic capacity (or the expectation of capacity) is given by the well-known formula [2]

$$
\bar{C}=E_{\mathbf{H}}\left\{\log _{2}\left(\operatorname{det}\left(\mathbf{I}_{N_{r}}+\frac{\rho}{N_{t}} \mathbf{H H}^{\mathrm{H}}\right)\right)\right\}
$$

In our study, we generated 50,000 realizations of $\mathbf{H}$ using the same fixed settings for the LoS matrix $\mathbf{H}_{\mathrm{L}}$ as previously. The entries of $\mathbf{H}_{\mathrm{W}}$ were assumed to follow the Rayleigh distribution and therefore are modeled as i.i.d complex variables with zero mean and unity variance. The main goal is to examine the effects of the $K$-factor on the ergodic capacity and for this reason we have plotted the corresponding cumulative distribution functions (cdfs) in Fig. 4.

For the specific full-rank geometry we stress out the monotonical capacity increase with the $K$-factor as well as the enhanced diversity performance (steeper cdf curves). This contradicts the common belief that higher $K$-factors, which induce dominant deterministic components, lead to higher correlation and thus lower capacity; in strong LoS conditions the proposed configuration significantly outperforms the conventional architectures. Our results are consistent with those presented in [6]-[8]. To demonstrate clearly this phenomenon we have also examined a conventional architecture with $0.5 \lambda$

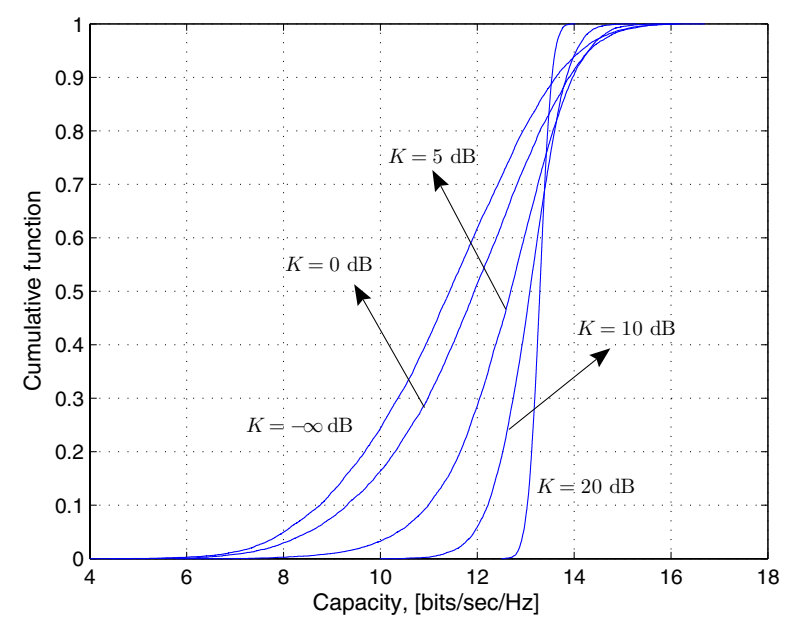

Fig. 4. Capacity cdfs of a full-rank stochastic MIMO channel for different values of the $K$-factor $(\rho=20 \mathrm{~dB})$.

inter-element distances at both ends. An inverse trend is now observed with the $K$-factor canceling off the MIMO advantages. In the limit $(K=-\infty \mathrm{dB})$ the LoS component vanishes and we end up with a pure i.i.d Rayleigh channel.

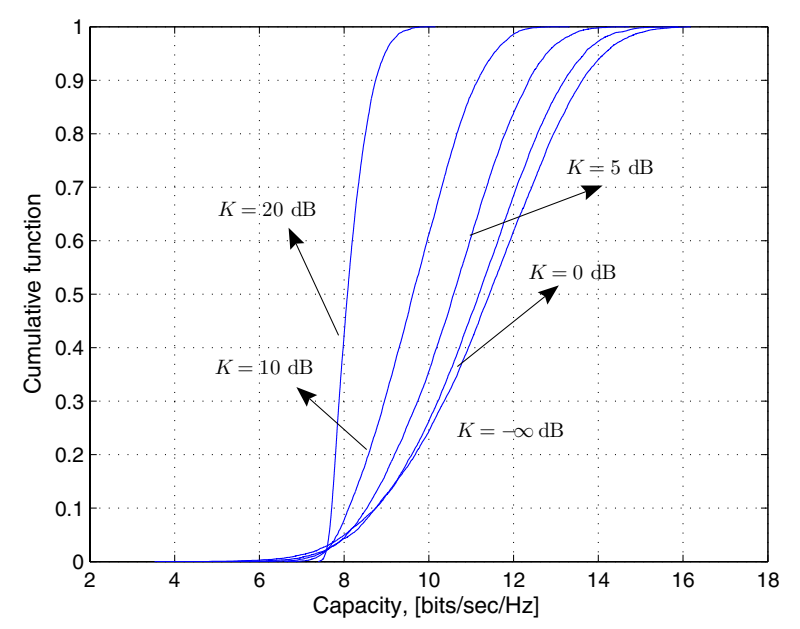

Fig. 5. Capacity cdfs of a conventional stochastic MIMO channel for different values of the $K$-factor $(\rho=20 \mathrm{~dB})$.

At a next stage, the dynamic evolution of capacity is studied again for different values of the $K$-factor and the obtained results are shown in Fig. 6. As the $K$-factor increases the dynamic range of capacity increases too; for $K \geq 10 \mathrm{~dB}$ the channel capacity is able to reach its maximum value and becomes more robust to displacements from optimum locations. On the other hand, for $K \leq 0 \mathrm{~dB}$ the system performance is unaffected by the car motion and small deviations from the ergodic Rayleigh capacity are noticed which implies that the benefits of LoS-optimized arrays are minimized. 


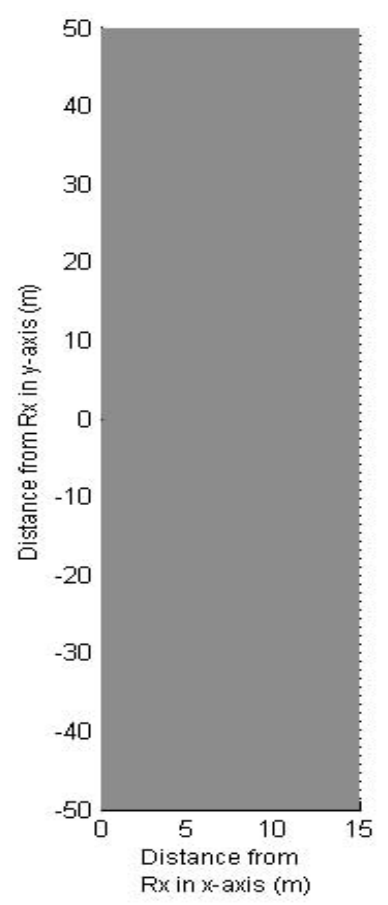

(a) $K=0 \mathrm{~dB}$

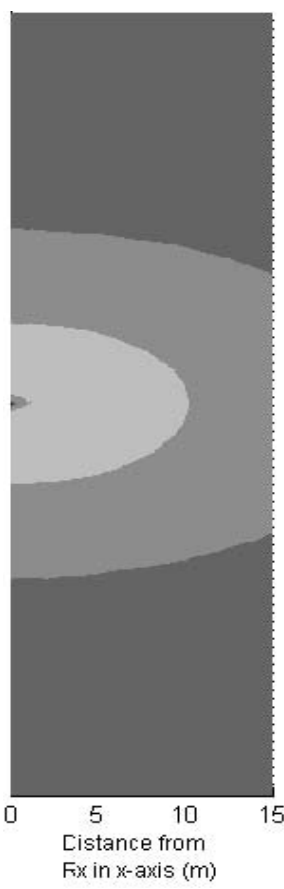

(b) $K=5 \mathrm{~dB}$

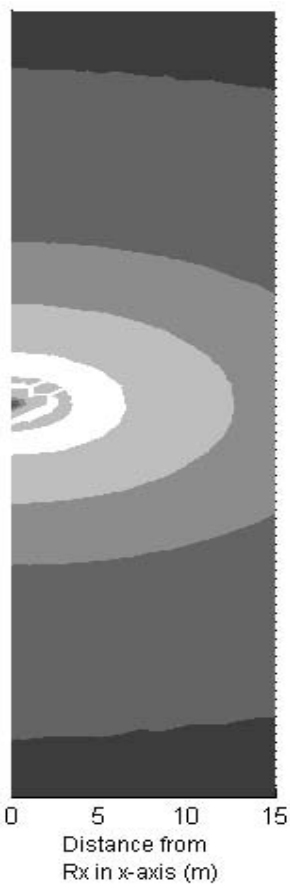

(c) $K=10 \mathrm{~dB}$

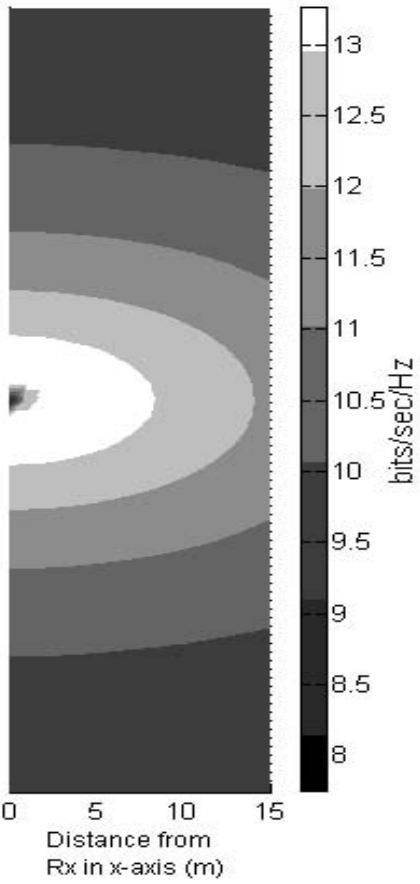

(d) $K=20 \mathrm{~dB}$

Fig. 6. Sensitivity of capacity to displacements on the $x-y$ plane for different values of the $K$-factor.

\section{CONCLUSION}

In this paper, we have examined the performance of a MIMO vehicle-to-roadside channel under LoS propagation. Contrary to common belief, LoS channels are not always rank deficient; in fact, they can deliver capacities much higher than Rayleigh (rich scattering) channels do, with appropriate positioning of the antenna arrays. A recently proposed criterion was revised for a specific propagation scenario and subsequently we tested its sensitivity to displacement and scattering effects. The considered scheme remains robust even when large displacements occur. The beneficial effects of the $K$ factor were validated and we concluded that the presence of dominant specular components stabilizes the link. To sum up, the proposed scheme seems appropriate for dedicated short range communications offering a high coverage and enhanced data rates at higher frequency bands.

\section{ACKNOWLEDGMENTS}

The authors would like to thank Dr. Ioannis Sarris from Mitsubishi Electric ITE-VIL, and formerly of Bristol University, for suggesting adaption of the methods presented in [6] and [7] to this specific scenario. They would like to acknowledge the support of the Scottish Funding Council for the Joint Research Institute with the Heriot-Watt University which is a part of the Edinburgh Research Partnership.

\section{REFERENCES}

[1] Task group p, "IEEE 802.11p Wireless Access for Vehicular Environment," http://grouper.ieee.org/groups/802/11/
[2] G. Foschini and M. Gans, "On Limits of Wireless Communication in a Fading Environment when using Multiple Antennas," Wireless Pers. Commun., vol. 6, no. 3, pp. 311-335, February 1998.

[3] D. P. McNamara, M. A. Beach, P. N. Fletcher, and P. Karlsson, "Capacity variation of indoor multiple input multiple output (MIMO) channels," IEE Electronics Letters, vol. 36, no. 24, pp. 2037-2038, November 2000.

[4] L. Cottatelluci and M. Debbah, "On the capacity of MIMO Rice Channels," in Proc. 42nd Allerton Conference, Illinois, USA, 2004.

[5] B. Badic and J. Choi, "Channel Capacity in Road-to-Vehicle MIMO Wireless Communications, in press Signal Processing for Wireless Communication (SPWC)", London, U.K., June 2007.

[6] I. Sarris and A. R. Nix, "Design and Performance Assessment of High-Capacity MIMO Architectures in the Presence of a Line-of-Sight Component," IEEE Transactions on Vehicular Technology, vol. 56, no. 4, pp. 2194-2202, July 2007.

[7] I. Sarris and A. R. Nix, "Design and Performance Assessment of Maximum Capacity MIMO Architectures in Line-of-Sight," IEE Proceedings in Communications, vol. 153, no. 4, pp. 482-488, August 2006.

[8] F. Bohagen, P. Orten, and G. E. Oien, "Construction and capacity analysis of high-rank line-of-sight MIMO channels," in Proc. IEEE Wirel. Commun. and Netw. Conf., March 2005, pp. 432-437.

[9] D. Gesbert, H. Bolcskei, D. A. Gore, and A. J. Paulraj, "Outdoor MIMO wireless channels: Models and performance prediction," IEEE Trans. Commun., vol. 50, no. 12, pp. 1926-1934, December 2002.

[10] P. Almers et al., "Survey of Channel and Radio Propagation Models for Wireless MIMO Systems," EURASIP Journal on Wireless Communications and Networking, pp. 1-19, vol. 2007.

[11] F. Rashid-Farrokhi, A. Lozano, G. Foschini, and R. Valenzuela, "Spectral efficiency of wireless systems with multiple transmit and receive antennas," in Proc. International Symposium on Personal, Indoor and Mobile Radio Communications (PIMRC), vol. 1, Sept. 2000, pp. 373-377. 